\title{
La creación sinfónica tras el III centenario del Quijote. El compositor Emilio Serrano y su obra de temática cervantina
}

\author{
Symphonic Compositions after the Third Centenary of El Quixote. The \\ Composer Emilio Serrano and his Cervantine Works
}

\author{
Beatriz Alonso Pérez-Ávila \\ Universidad de Oviedo \\ beatrizalonsoperezavila@gmail.com
}

\begin{abstract}
RESUMEN
Emilio Serrano y Ruiz (1850-1939) es una figura de relevancia fundamental para comprender la música española de finales del siglo XIX y principios del XX. A pesar de que su labor compositiva destaca por presentar obras de envergadura en todos los géneros, Serrano es recordado exclusivamente como autor lírico. Sin embargo, recientes investigaciones muestran un catálogo sinfónico de primera línea en el que destacan dos poemas sinfónicos dedicados a la inmortal obra de Cervantes, compuestos bajo el fervor cervantino de la celebración del III centenario (Alonso, 2015). En este artículo se presentan La primera salida de Don Quijote de la Mancha y Don Quijote de la Mancha, canto segundo. Los molinos de viento, revisándose las interpretaciones en concierto, estableciendo las características del lenguaje compositivo del autor y, finalmente, se sitúan en el contexto de su época.
\end{abstract}

Palabras clave: Emilio Serrano, poemas sinfónicos, III centenario, sinfonismo español, música cervantina.

\begin{abstract}
Emilio Serrano y Ruiz (1850-1939) is an essential figure to understand the music composed in Spain in the late $19^{\text {th }}$ and early $20^{\text {th }}$ centuries. Although his oeuvre stands out for including important works in all genres, Serrano is exclusively remembered as a lyrical composer. However, recent studies reveal a first-class symphonic catalogue, where two symphonic poems devoted to Cervantes' immortal works stand out, written under the Cervantine fervor of the celebration of the third centenary of El Quixote (Alonso, 2015). This paper deals with La primera salida de Don Quijote de la Mancha and Don Quijote de la Mancha, canto segundo. Los molinos de viento, examining concert performances and establishing the characteristics of the author's composing style, to finally place them in the context of their time.
\end{abstract}

Key words: Emilio Serrano, Symphonic Poems, $3^{\text {rd }}$ Centenary, Spanish Symphonies, Cervantine Music. 


\section{BeAtriz Alonso PÉrez-Ávila}

Alonso Pérez-Ávila, B. (2017). La creación sinfónica tras el III centenario del Quijote. El compositor Emilio Serrano y su obra de temática cervantina. Cuadernos de Investigación Musical, 2, 3958.

doi: http://dx.doi.org/10.18239/esmusic_2017.02.1548

\section{INTRODUCCIÓN}

Emilio Serrano y Ruiz (1850-1939) forma parte de la generación de compositores nacidos a mediados del siglo XIX. Su personalidad musical se desarrolló fundamentalmente en tres vertientes: como compositor, como docente y como miembro destacado de distintas instituciones y asociaciones (Cortizo, 1990). Estudió en el Conservatorio de Madrid y fue pensionado de mérito en Roma. En el centro madrileño ejerció la docencia durante más de cuarenta años, destacándose como catedrático de composición. Bajo su tutela se formaron músicos como Conrado del Campo o Julio Gómez. Paralelamente fue también Maestro de Cámara de la Infanta durante más de dos décadas. Entre las distintas instituciones a las que perteneció destaca la Real Academia de Bellas Artes de San Fernando y el Círculo de Bellas Artes. También fue director artístico del Teatro Real y del Instituto Filarmónico ${ }^{1}$.

Como compositor se le recuerda por ser uno de los grandes defensores de la ópera nacional, a la que dedicó su discurso de ingreso en la Real Academia (Serrano, 1901). Estrenó cuatro óperas en el Teatro Real y una quinta en el Teatro Colón. Sin embargo, su producción zarzuelística está compuesta exclusivamente por cinco zarzuelas. A pesar de que se recuerde a Serrano por su relación con el género lírico, en realidad compuso obras de todos los géneros, con un repertorio amplio en el que destacan obras como el Concierto para piano y orquesta (1895), la Elegía a la muerte de Fernando Villamil (1915) o La Primera Salida de Don Quijote de la Mancha (1912).

El tema del Quijote ha sido fuente de inspiración de numerosas creaciones artísticas desde la publicación de las dos partes de la novela cervantina. Sin embargo, es en torno al tercer centenario de la publicación de la primera parte de Don Quijote de La Mancha de Miguel de Cervantes cuando se observa un aumento significativo en el número de obras musicales con referencias a esta obra. El interés suscitado en este tercer centenario no es comparable con los siguientes centenarios - de la segunda parte y de la muerte de Cervantes-, sin embargo, se podría decir que esta primera efeméride sirvió de acicate compositivo y este interés ya no se volvió a apagar. Los motivos que justifican esta súbita fascinación parecen claros en cuanto "se observa una intención claramente política en la utilización de esta fecha como reafirmación nacional, como forma de contrarrestar el ambiente de pesimismo que instaló entre los intelectuales y el pueblo español tras la pérdida de las colonias americanas" (Presas, 2007: 285).

\footnotetext{
${ }^{1}$ Emilio Serrano será uno de los impulsores de esta institución educativa, que pretendía ser un centro de enseñanza musical paralelo al Conservatorio. Sin embargo, la pensión de mérito para estudiar en Roma concedida a Emilio Serrano hará que deje su puesto a Ruperto Cancio (García, 2016).
} 
LA CREACIÓN SINFÓNICA TRAS EL III CENTENARIO DEL QUIJOTE. EL COMPOSITOR EMILIO SERRANO Y SU OBRA DE TEMÁTICA CERVANTINA

Emilio Serrano, como miembro activo de las más importantes instituciones culturales del momento, se implicó directamente en los distintos homenajes que se fueron preparando para el centenario de la publicación de la primera parte del Quijote. Fue miembro de la comisión que preparó el concierto homenaje en el Conservatorio, junto a Bretón, Valderrama, Mendoza y Fontanilla², que se celebró el día 18 de mayo de 1905³ y también formó parte de la comisión designada por la sección de música de la Real Academia de Bellas Artes de San Fernando para organizar otro concierto homenaje que tuvo lugar el día 10 de mayo en el Teatro Real ${ }^{4}$. Esta comisión estuvo formada por Fernández Caballero, Bretón y Serrano ${ }^{5}$, quienes quedaron encargados de organizar actividades en torno al Quijote 6 . Este concierto supone un hito por la interpretación de piezas sinfónicas sobre temática cervantina (Presas, 2007: 301) ya que previamente tan sólo encontramos una pieza de juventud de Chapí, Combate de Don Quijote con las ovejas, de 1868. Así, a partir del año 1905, la composición orquestal se sucederá a lo largo de todo el siglo $\mathrm{XX}$, con picos de producción más pronunciados, normalmente coincidentes con otras celebraciones cervantinas. Uno de los ejemplos es la obra de Jesús Guridi Una aventura de Don Quijote, de 1915, que recibió el segundo premio en el concurso para obras sinfónicas del Círculo de Bellas Artes ${ }^{7}$, en el que Serrano ejerció de presidente del jurado (Martínez el Fresno, 1999: 118).

\section{LOS POEMAS SINFÓNICOS DE EMILIO SERRANO DE TEMÁTICA CERVANTINA}

En los primeros años del siglo XX, Serrano ya era un compositor maduro. De este período datan sus dos poemas sinfónicos de temática quijotesca ${ }^{8}$, La primera salida de Don Quijote de La Mancha y Don Quijote, Canto segundo: Los molinos de viento.

\footnotetext{
2 Acta de la sesión celebrada por el Claustro del Conservatorio de Música y Declamación el día 20 de enero de 1905.

3 Véase: "En honor de Cervantes" (1905, 19 de mayo). El Globo, 19 de mayo de 1905, p.2; "En el Conservatorio" (1905, 19 de mayo). El Liberal, p. 2 y "En el Conservatorio" (1905, 20 de mayo) Diario Oficial de Avisos de Madrid, p. 3.

${ }^{4}$ Véase: "La función del Real suspendida". El Imparcial, 9 de mayo de 1905, p. 1.

${ }^{5}$ Acta de la sesión celebrada el 13 de febrero de 1905. RABASF [Sig. 3/313].

${ }^{6}$ Acta de la sesión celebrada el 30 de enero de 1905. RABASF [Sig. 3/313].

7 Véase: "Un concurso. Música española". $A B C, 12$ de junio de 1915, p. 15; "Músicos y conciertos". ABC, 23 de octubre de 1915, p. 20.

8 De La primera salida de Don Quijote se conserva una copia en la Biblioteca Musical "Víctor Espinós" de Madrid [Sig. QJ74] y su reducción para piano [Sig. QJ73], bajo el nombre de Don Quijote y con fecha de 1920. En la biblioteca de la Fundación Juan March se conserva una copia con una dedicatoria manuscrita a Julio Gómez [Sig. M/418/A] "A mi querido amigo y discípulo Julio Gómez en testimonio de profundo cariño, Madrid 1908"; y otra copia [Sig. M/416/A] encuadernada junto a otras piezas sinfónicas del autor: La fioraia, El tiovivo, Una copla de la jota y Don Quijote de La Mancha, Canto segundo. Los molinos de viento. En el caso del poema sinfónico Don Quijote, Canto segundo. Los molinos de viento, conservamos la copia anteriormente señalada [Sig. M/416/A] en la Fundación Juan March y la partitura manuscrita original en la biblioteca del Conservatorio Superior de Madrid [Sig. 4/405].
}

Cuadernos de Investigación Musical, 2017, junio, nº 2, págs. 39-58.

ISSN: 2530-6847 
BEATriz ALONSO PÉREZ-Ávila

\begin{tabular}{|c|c|c|}
\hline & $\begin{array}{c}\text { La primera salida de Don Quijote de la } \\
\text { Mancha }\end{array}$ & $\begin{array}{c}\text { Don Quijote, Canto segundo: Los molinos de } \\
\text { viento }\end{array}$ \\
\hline Género & Poema sinfónico. & Poema sinfónico. \\
\hline $\begin{array}{c}\text { Plantilla } \\
\text { orquestal }\end{array}$ & $\begin{array}{c}\text { vn1, vn2, va, vc, cb, flt, fl, ob, crn, cl, } \\
\text { cl bajo, fg, tp, tpt, tbn, tu, tim, bo, } \\
\text { plat y ar. }\end{array}$ & $\begin{array}{c}\text { vn1, vn2, va, vc, cb, flt, fl, ob, crn, cl } \\
\text { (Sib), cl (La), fg, tp (Fa), tpt (Do), tbn, } \\
\text { tu, tim, ar. }\end{array}$ \\
\hline $\begin{array}{c}\text { Otros } \\
\text { datos }\end{array}$ & $\begin{array}{c}\text { Dedicado a D. Julio Gómez. } \\
\text { Partitura manuscrita fechada en } \\
\text { "Cercedilla, 29-VIII-1905". } \\
\text { Estrenada el 30 de mayo de 1912 en } \\
\text { el Teatro Español". }\end{array}$ & $\begin{array}{c}\text { Inédito. } \\
\text { En la copia manuscrita conservada en } \\
\text { la biblioteca del Conservatorio de } \\
\text { Madrid aparece como fecha de } \\
\text { composición (apuntes) el día "8-VIII- } \\
\text { 1905", añadiéndose que la } \\
\text { instrumentación se acabó con fecha } \\
\text { de "16-IV-1908". }\end{array}$ \\
\hline
\end{tabular}

Tabla 1. Datos sobre los poemas sinfónicos cervantinos de Serrano.

A pesar de que Serrano ya había compuesto varios poemas sinfónicos, ciertamente es sorprendente que no intentase utilizar El Quijote para realizar una nueva ópera. En estos años, Serrano ya había acabado de componer La maja de rumbo junto a Carlos Fernández Shaw para el Teatro Lírico de Chapí (G. Iberni, 1995: 332). Incluso la prensa parecía esperar antes una obra teatral que una sinfónica, como señala este artículo aparecido en $E l$ Globo, que describe una visita a la casa del Maestro en Cercedilla:

El maestro Serrano trabaja mucho, y en la actualidad, compone un poema inspirado en asuntos del Quijote, que como obra suya, ha de ser genial y estimada como otro noble esfuerzo en pro de la ópera española, por cuyo engrandecimiento batalla el ilustre compositor $^{10}$.

Consideramos que fueron varios los motivos por los que el autor utilizó el género instrumental. Por un lado, para él fueron fundamentales los actos de celebración del año 1905, en los que por primera vez tuvieron cabida piezas de temática cervantina exclusivamente instrumentales. Por otro lado, probablemente consideró que, por medio de la música instrumental, podía recrear más libremente su propia visión del Quijote que si se ceñía a un cuadro dramático determinado ${ }^{11}$. Cabe recordar que Serrano consideraba que el camino más breve y acertado para llegar a una forma de perfecta belleza musical era, desde

\footnotetext{
9 Véase: "Círculo de Bellas Artes, concierto Larregla" (1912, 21 de abril). El País, p. 2.

${ }^{10}$ Véase: Marton e Izaguirre, J. (1905, 26 de agosto). "Cercedilla". El Globo, p. 2. Tras el nombre del autor, lleva la siguiente inscripción: "Cercedilla, 22 Agosto 1905”.

${ }^{11}$ Aunque posteriormente habrá auténticos casos de evocación, como será el caso de Óscar Esplá (Nommick, 2007: 214).
} 
LA CREACIÓN SINFÓNICA TRAS EL III CENTENARIO DEL QUIJOTE. EL COMPOSITOR EMILIO SERRANO Y SU OBRA DE TEMÁTICA CERVANTINA

el punto de vista técnico, el puramente sinfónico ${ }^{12}$. Por último, la elección de Serrano de la temática cervantina no sólo se debe a la influencia de los actos de conmemoración del centenario, sino que encaja perfectamente con el pensamiento del compositor, quien, al igual que la mayoría de sus compañeros de generación, es profundamente nacionalista y busca en la utilización de símbolos históricos o míticos la grandeza perdida, consiguiendo así la España musical soñada. Serrano ya se había distinguido por escoger para sus óperas temas históricos, como Juana la Loca o Gonzalo de Córdoba. Significativo es el caso del estreno de su ópera Gonzalo de Córdoba, acaecido en diciembre de 1898, en el que, a juzgar por las críticas de la época ${ }^{13}$, se percibe en el autor una auténtica necesidad por identificarse con un pasado glorioso que hiciese olvidar las dolorosas derrotas y negar la cruda evidencia del presente del país. Con el Quijote, la generación del 98, sobre todo Unamuno y Azorín, llevan esta idea aún más lejos para imaginar un mito político. Es una forma de resarcirse del desastre a través de la glorificación del gran mito que es el Quijote (Lolo, 2010: 86), que trasciende los confines del libro y se convierte en uno de los grandes iconos que identifican la imagen de España en el exterior.

\subsection{LA PRIMERA SALID A DE DON QUIJOTE DE LA MANCHA}

"Donde, a través de una técnica moderna de libérrima estructura, fulgura la inspiración castiza de la musa popular” (Manrique de Lara, 23/05/1915: 36).

Este poema sinfónico es una de las piezas más interpretadas de Emilio Serrano. En la Biografía de Subirá se recogen las notas al programa del concierto de estreno de esta obra que, con una intención claramente didáctica, guían al oyente y ofrecen datos valiosos sobre la intención del compositor:

Una de las cualidades más preciadas del inmortal poema de Cervantes es la de que en cada capítulo, en cada fragmento, está, por maravillosa facultad sintética del genio, comprendida toda la idea filosófica del autor. Y más que en ningún otro trozo, acaece esto en los cinco primeros capítulos de la primera parte, en los cuales se narra la primera salida del hidalgo. Allí se dibuja ya perfectamente su personalidad, cuya nota esencial y característica es el amor. Don Quijote, como dice Turgueneff ${ }^{14}$, cree indigno de él vivir para sí mismo, cuidarse de su persona; vive constantemente fuera de sí, para los demás, para sus hermanos; sirve para extirpar lo malo, para combatir a las fuerzas enemigas del hombre: gigantes, encantadores, opresores de los endebles. No hay en él rastro de egoísmo; jamás piensa en sí; es todo sacrificio; cree, tiene buena fe y marcha siempre adelante sin mirar atrás.

\footnotetext{
12 Véase: Serrano Ruiz, E. Conferencia sobre el Teatro Real y el de la Zarzuela. Cuartilla sobre el Teatro Real con correcciones autógrafas. Interviú sobre música teatral y sinfónica. Manuscrito no publicado presente dentro de los documentos sobre la vida y la obra de Emilio Serrano Ruiz, pp. 20 y 21. RABASF [Sig. 6/82/1].

13 A modo de ejemplo, recogemos "¡En España no hay desdichas, no ha habido guerras, no se pone el sol en nuestros dominios, tenemos, en fin, un músico como Emilio Serrano capaz de escribir una ópera como Gonzalo de Córdoba, que puede salir a torneo como su protagonista segura de vencer!". De Lara, A. D. (1898, 7 de diciembre). "Teatro Real, Gonzalo de Córdoba". La Correspondencia militar, p. 2.

${ }_{14}$ Se refiere el autor a Ivan Turgueniev (1818-1883), autor ruso que escribió el ensayo Hamlet y Don Quijote, basándose en una conferencia pronunciada en 1860 en San Petersburgo.
}

Cuadernos de Investigación Musical, 2017, junio, nº 2, págs. 39-58. 


\section{BeAtriz Alonso Pérez-Ávila}

En la primera salida de Don Quijote está sintetizada toda la obra: sus imperecederos ideales, los choques que se producen con la realidad corriente y prosaica de la vida, su inconmovilidad ante ellos y la afirmación eterna del amor inmortal. Ésta es la idea madre del poema de Serrano, y por eso puede, como aquellos primeros capítulos, considerarse síntesis de la vida de Don Quijote (Subirá, [s.f.]: 5-6) ${ }^{15}$.

\begin{tabular}{|c|c|}
\hline Fecha & Observaciones \\
\hline $30-\mathrm{V}-1912$ & Teatro Español, dirigida por Emilio Serrano. \\
\hline 31-XII-1920 & Teatro del Centro, dirigido por el maestro Lassalle ${ }^{16}$. \\
\hline $23-\mathrm{I}-1921$ & Teatro del Centro, dirigido por el maestro Lassalle ${ }^{17}$. \\
\hline 4-III-1921 & $\begin{array}{l}\text { Circo Price por la orquesta Filarmónica }{ }^{18} \text { dirigida por Pérez Casas. } \\
\text { Concierto patrocinado por el Círculo de Bellas Artes dentro de los } \\
\text { Conciertos Populares. }\end{array}$ \\
\hline $9-\mathrm{X}-1921$ & Teatro del Centro, dirigido por el maestro Lassalle ${ }^{19}$ \\
\hline 8 о 9-I-1924 & $\begin{array}{l}\text { Orquesta Sinfónica portuguesa, noveno concierto en el Teatro de San } \\
\text { Luis, dirigido por Lassalle }{ }^{20} \text {. }\end{array}$ \\
\hline 27-XI-1926 & Palacio de la Música, dirigido por el maestro Lassalle ${ }^{21}$. \\
\hline 12-XII-1929 & $\begin{array}{l}\text { Palacio de la Música, dirigido por el maestro Lassalle. Este concierto se } \\
\text { organiza como homenaje a la figura de Serrano }\end{array}$ \\
\hline
\end{tabular}

Tabla 2. Interpretaciones de La primera salida de Don Quijote de la Mancha ${ }^{23}$.

15 Véase: Subirá, J. Biografía del compositor Emilio Serrano y su época. Manuscrito no publicado, pp. 5-6. Biblioteca Nacional de Catalunya [P2/3 C63/81].

16 Véase: "Diversiones públicas" (1920, 30 de diciembre). La Época, p. 4; "Informaciones musicales, en el centro. La orquesta Lassalle" (1921, 1 de enero). $A B C$, p. 22.

${ }^{17}$ Véase: "Concierto Matinal de la orquesta Lassalle" (1921, 25 de enero). $A B C$, p. 21.

18 "Informaciones musicales, Orquesta Filarmónica" (1921, 3 de marzo). $A B C$, p. 22.

${ }_{19}$ Véase: El licenciado Pontejos (1921, 8 de noviembre). "Los conciertos íntimos en el Centro", La Ilustración Española y Americana, p. 11; "Informaciones y noticias musicales. La orquesta Lassalle" (1921, 8 de octubre). $A B C$, p. 8; Forns, J. (1921, 11 de octubre). "Primer concierto Lasalle", Heraldo de Madrid, p. 5.

${ }^{20}$ No tenemos clara la fecha exacta de este concierto, pero no pudo ser posterior al día 9. Por otra parte, hay que señalar que no se trata de la Orquesta Sinfónica Portuguesa actual, que fue creada en 1993. Esta era la orquesta dirigida por el Maestro Blanch y se trataba del $9^{\circ}$ concierto de la temporada. El concierto tuvo cierta importancia y acudió el presidente de la República y el Jefe de Estado portugués, interpretándose también $A$ Patria, de Vianna la Motta. Véase: "Los éxitos del maestro Lassalle" (1924, 9 de enero). La Correspondencia de España, p. 8; "El maestro Lassalle, en Lisboa" (1924, 9 de enero). La Voz, p. 1; y "Notas Varias de Portugal" (1924, 10 de enero). $A B C$, pp. 20-21.

${ }^{21}$ Véase: "Informaciones musicales, los conciertos de la Orquesta Lassalle" (1926, 28 de noviembre). $A B C, \mathrm{p}$. 36.

22 Véase: (1929, 23 de octubre). $A B C$, p. 49; (1929, 10 de diciembre). $A B C$, p. 50; (1929, 11 de diciembre). $A B C$, p. 45; "Los alumnos de Emilio Serrano" (1929, 8 de diciembre). $A B C$, p. 33; (1929, 12 de diciembre). $A B C$, p. 38; y (1929, 13 de diciembre). $A B C$, p. 43; A. M. C. (1929, 14 de diciembre). "Los conciertos de la Orquesta Lassalle y el homenaje al maestro Serrano". $A B C$, pp. 44 y 45; "Homenaje a D. Emilio Serrano" (1929, 14 de diciembre). El Imparcial, p. 6.

${ }^{23}$ En un avance del periódico El Sol se señala que, en el Teatro Principal de Valencia, habrá un concierto sinfónico dedicado a la música española en el que se interpretarán piezas de Falla, Ernesto Halffter, Emilio Serrano, Chávarri, Paláu, Giner, Gómez y Cuesta. No hemos encontrado más referencias a este concierto ni a qué pieza de Serrano se refiere, pero dado que La primera salida de Serrano era una obra del repertorio de la orquesta cabe suponer que fue la pieza que tocaron. Véase: "La vida musical" (1923, 13 de mayo). El Sol, p. 2. Por otra parte, tenemos una carta de Jesús Arámbarri a Julio Gómez guardada en la biblioteca de la Fundación Juan March en la que le solicita la partitura de la Primera Salida para poder interpretarla. Aunque de momento no hemos encontrado indicios de que llegase a hacerlo. Fundación Juan March [Sig. $\mathrm{M} / \mathrm{AE} / \mathrm{ARA} / 10]$. 
LA CREACIÓN SINFÓNICA TRAS EL III CENTENARIO DEL QUIJOTE.

EL COMPOSITOR EMILIO SERRANO Y SU OBRA DE TEMÁTICA CERVANTINA

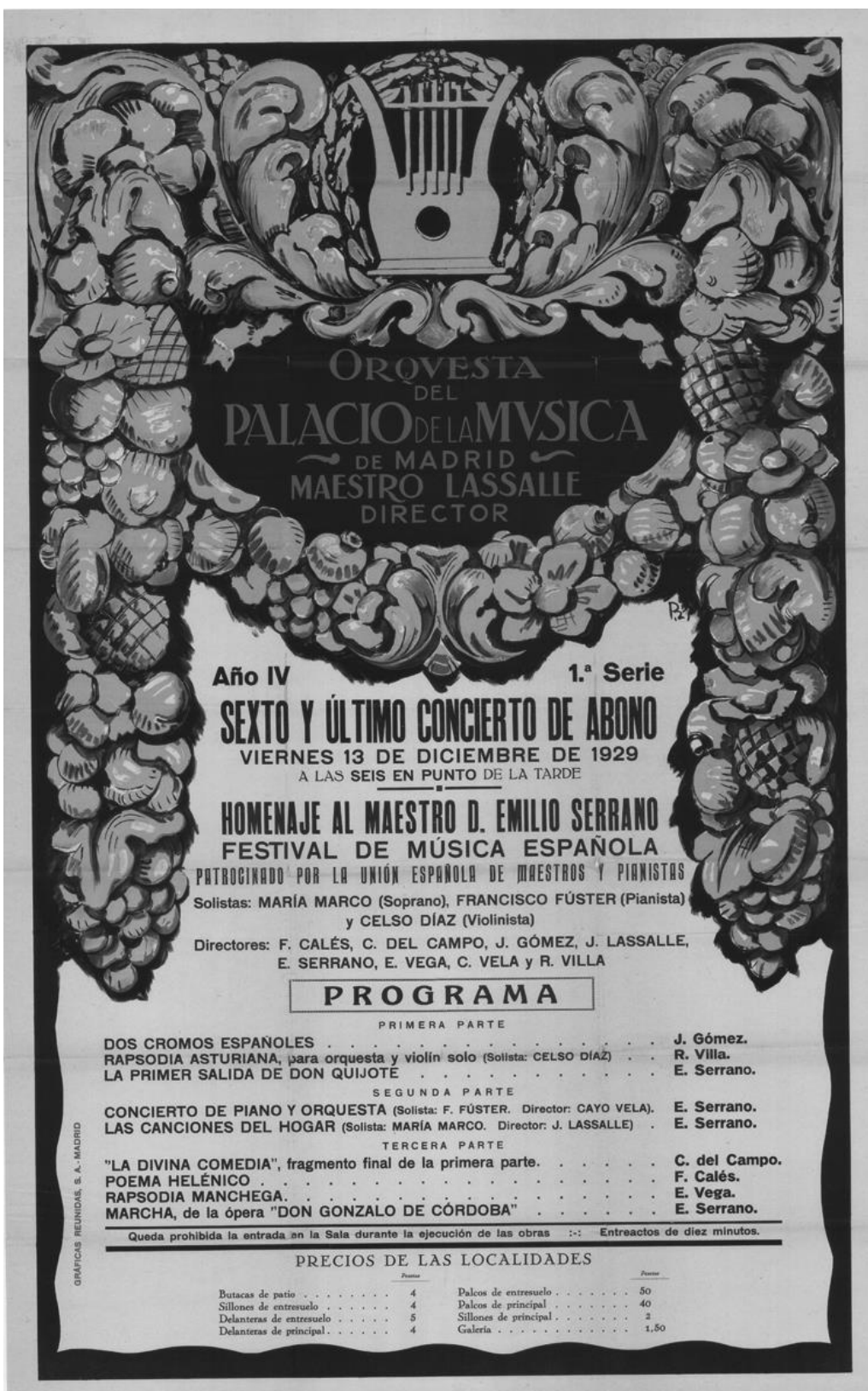

Fig 1. Cartel del concierto del día 12 de diciembre de 1929

(Biblioteca de la Fundación Juan March) 


\section{BEATRIZ Alonso PÉREZ-Ávila}

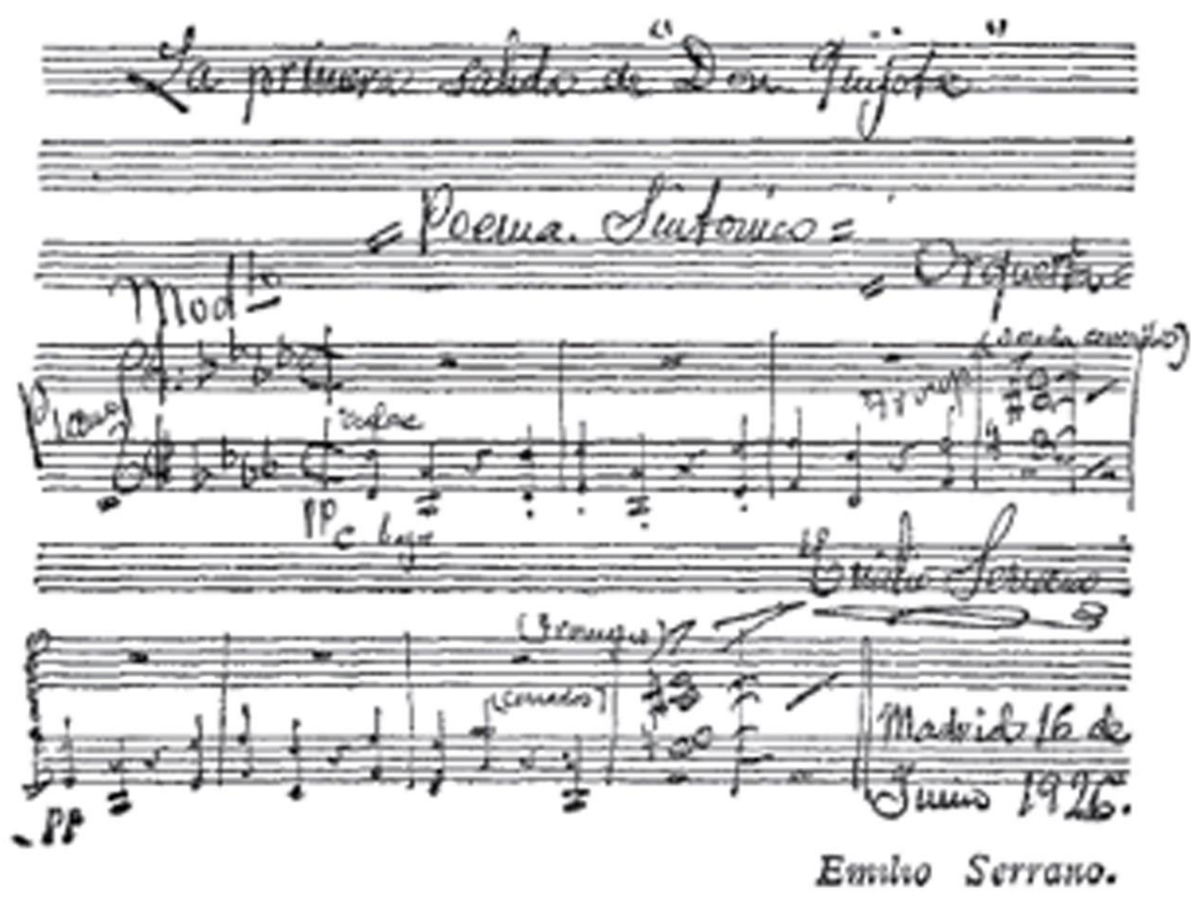

Fig. 2. Comienzo del arreglo para piano, aparecido en el diario $A B C^{24}$ firmado por Serrano, a modo de autógrafo musical de regalo a los lectores.

Con respecto a las fechas de composición y estreno, llama poderosamente la atención el hecho de que, a pesar de ser una obra compuesta al calor de las celebraciones del III centenario, no se hubiese estrenado en este año 1905, momento en el que se presupone que una obra dedicada al Quijote podría tener más oportunidades de ser interpretada. Por ello, y a pesar de que se compuso después de algunos de los conciertos sinfónicos importantes dedicados a la genial obra cervantina, no debería de haber sido tan complicado su estreno como parece que fue. Tuvo que esperar Serrano a un concierto dedicado a su figura para poder oír por primera vez esta obra. Por otra parte, a pesar de ser Serrano uno de los impulsores de los Conciertos Populares del Círculo de Bellas Artes, este poema sinfónico no se programa en ellos hasta 1921, y tan sólo en una ocasión. El poema de Serrano sin duda era comparable al de Tomás Bretón titulado Los Galeotes (1905), a pesar de que la obra del salmantino está perdida en la actualidad ${ }^{25}$, cabe suponer que el lenguaje musical de ambas obras fuese semejante (Cuadrado, 2010: 269). Sin embargo, la pieza de Bretón fue interpretada en tres ocasiones por la Orquesta Filarmónica (Cuadrado, 2010:275), mostrando que la influencia de Bretón sobre la programación de los Conciertos Populares debía de ser mayor que la de Serrano.

\footnotetext{
24 Véase: (1927, 29 de mayo). $A B C$, p. 27.

${ }^{25}$ No se ha localizado la partitura y para acercarnos a cómo pudo haber sido tenemos las críticas de la época (Sánchez, 2002: 375-376).
} 
LA CREACIÓN SINFÓNICA TRAS EL III CENTENARIO DEL QUIJOTE.

EL COMPOSITOR EMILIO SERRANO Y SU OBRA DE TEMÁTICA CERVANTINA

Formalmente, el poema sinfónico está dividido en dos cantos. Ambos comienzan con unas indicaciones programáticas. El primer canto presenta la siguiente inscripción: "Y así, sin dar parte a persona alguna de su intención”, que sitúa la obra en el comienzo del capítulo II de El Quijote. El programa de mano conservado realiza una comparación de la obra cervantina con la obra de Serrano, mostrando así la intención descriptiva del autor y situándonos dramáticamente a lo largo de todo el poema.

\begin{tabular}{|c|c|c|c|c|}
\hline & A & $\mathrm{B}$ & $\mathrm{C}$ & $\mathrm{D}$ \\
\hline \multirow[t]{4}{*}{ Compases } & \multirow[t]{2}{*}{ cc. $1-27$} & cc. $28-110$ & \multirow[t]{2}{*}{ cc. $132-162$} & cc. $163-179$ \\
\hline & & cc. $180-245$ & & сc. $277-289$ \\
\hline & \multirow[t]{2}{*}{ cc. $111-13126$} & cc. $290-314$ & \multirow[t]{2}{*}{ cc. $246-276$} & \multirow[t]{2}{*}{ cc. $315-327$} \\
\hline & & $\begin{array}{l}\text { cc. } 328-355 \text {, fin del } \\
\text { canto }\end{array}$ & & \\
\hline \multirow[t]{4}{*}{ Tonalidades } & \multirow[t]{2}{*}{ Do M, Sib M } & $\begin{array}{l}\text { Mib M, Lab M, Mib M, } \\
\text { Lab M, Mib M }\end{array}$ & $\begin{array}{l}\text { Mi M, Fa\# m, } \\
\text { Mi M, La m, Re } \\
\text { M, Mib M }\end{array}$ & $\begin{array}{l}\text { Sol m, Sib } M \text {, } \\
\text { Sol m, Sib } M \text {, } \\
\text { Sol m }\end{array}$ \\
\hline & & Sol M & $\begin{array}{l}\text { Mib M, Fa m, } \\
\text { Mib M, Fa m, } \\
\text { Sol b M, Sol b } \\
\text { m, Mi M }\end{array}$ & Mi m \\
\hline & \multirow[t]{2}{*}{ Mib M, Sib M } & Mi M & & \multirow[t]{2}{*}{ Sol m, Mi M } \\
\hline & & Mi M & & \\
\hline \multirow[t]{4}{*}{ Compás } & \multirow[t]{2}{*}{$2 / 2$} & $3 / 4$ & \multirow[t]{2}{*}{$2 / 2$} & $4 / 4$ \\
\hline & & $3 / 4$ & & $4 / 4$ \\
\hline & \multirow[t]{2}{*}{$2 / 2$} & $3 / 4$ & \multirow[t]{2}{*}{$2 / 2$} & \multirow[t]{2}{*}{$4 / 4$} \\
\hline & & $3 / 4$ & & \\
\hline \multirow[t]{4}{*}{ Tempi } & \multirow[t]{4}{*}{ Moderato } & Allegro,Scherzando, Presto & \multirow[t]{2}{*}{ Maestoso } & Moderato \\
\hline & & Allegro, Presto & & No se especifica \\
\hline & & Presto & \multirow[t]{2}{*}{ Maestoso } & \multirow[t]{2}{*}{ Molto moderato } \\
\hline & & Presto & & \\
\hline
\end{tabular}

Tabla 3. Estructura general del $1^{\text {er }}$ canto de La primera salida de Don Quijote de la Mancha.

${ }^{26}$ Existen diferencias de compases entre partitura orquestal y la reducción para piano. A lo largo del análisis utilizaremos los números de compás de la reducción para piano, pero hay que tener en cuenta que la partitura orquestal contiene un compás menos. Fundación Juan March [Sig. QJ73 y QJ74].

Cuadernos de Investigación Musical, 2017, junio, nº 2, págs. 39-58. 


\section{BeAtriz Alonso PÉrez-Ávila}

La primera sección, A, se caracteriza por un marcial pizzicatto en pianissimo de las cuerdas graves que se contrapone con notas largas en las trompas. Los pizzicatti que se realizan sobre este compás de 4/4 juegan con los silencios de la tercera parte del compás, incidiendo poderosamente en la parte fuerte. Si seguimos la novela cervantina, observamos que lo que describe Serrano es la partida de su casa.

Tras esos pirzicatti, surge el solo del oboe, sobre una nota tenida en piano en la cuerda, que puede interpretarse como ese pensamiento que le hace dudar a don Quijote. Esta breve melodía, de apenas cuatro compases, en Sib Mayor, la repetirá en eco el corno inglés, dándonos así idea de la importancia que se les dará a los instrumentos de viento madera a lo largo de toda la obra.

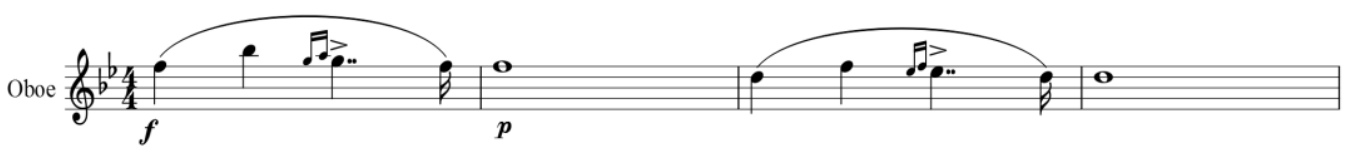

Ejemplo musical 1. Compases 19-22 del oboe del $1^{\text {er }}$ canto de La primera salida de Don Quijote ${ }^{27}$.

La sección $\mathrm{B}$, en 3/4 aligera el tempo y añade color orquestal con la utilización del arpa. El compás ternario modifica ya los acentos y, además, contrapone compases ternarios con binarios, rasgo que se convertirá en una constante a lo largo de todo el canto primero.

El programa continúa describiendo esta sección, señalando que:

Comienza el compositor dándonos la sensación de tranquilidad y silencio de aquella memorable madrugada del día (que era uno de los calurosos del mes de julio) en que Don Quijote, con sigilo y calladamente, después de haberse armado de todas las armas, por la puerta falsa de un corral salió al campo, con grandísimo contento y alborozo de ver con cuánta facilidad había dado principio a su buen deseo. El pizzicicato en la cuerda, los acordes de las trompas, consiguen que penetremos en el misterio nocturno. Un luminoso diseño del oboe, repetido melancólicamente por el corno inglés, nos hace presentir el día; después una idea aérea, en $3 / 4$, nos pone en contacto con el mundo imaginario que puebla el pensamiento de Don Quijote (Subirá, [s.f.]: 5-6].

La sección C, en, Maestoso, nos mantiene ligeramente en la sonoridad del inicio, con la contraposición entre trompas y cuerda, que se mantiene en pizzicatto. La sección D acaba en un pasaje de gran fuerza melódica, de factura claramente romántica. Puede ser que Serrano pensase en este tema como el tema de Dulcinea, aunque no lo indicase en la partitura. El fraseo no es regular, presentando una frase de 9 compases. 27 Edición propia partiendo de la fuente presente en la Biblioteca Musical "Víctor Espinós" de Madrid [Sig.
QJ74]. 
LA CREACIÓN SINFÓNICA TRAS EL III CENTENARIO DEL QUIJOTE. EL COMPOSITOR EMILIO SERRANO Y SU OBRA DE TEMÁTICA CERVANTINA
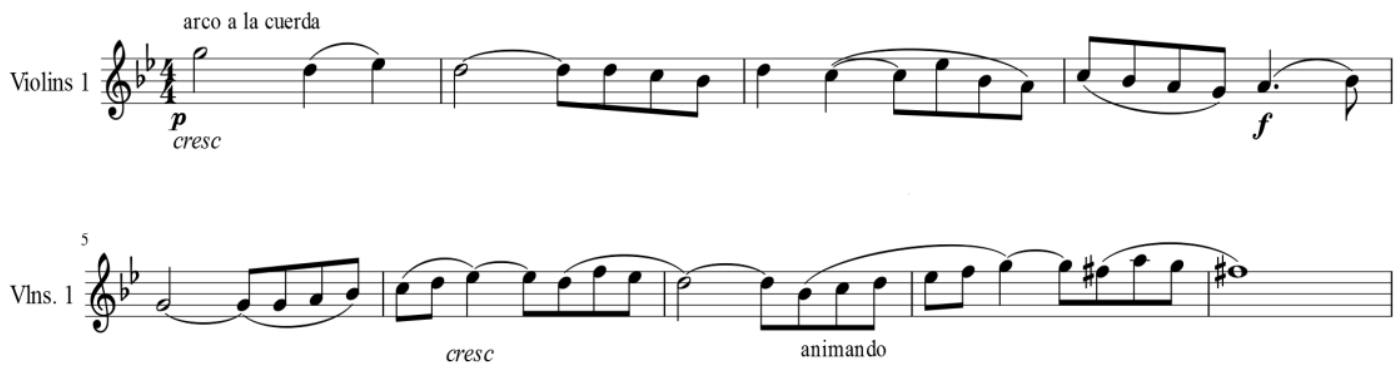

Ejemplo musical 2. Compases 163-171 de los violines I y II del 1er canto de La primera salida de Don Quijote de la Mancha.

Sucesivamente se presentan la idea caballeresca, noble y varonil, que dicen las trompas sobre un marcial pizzicato de toda la cuerda y la apasionada melodía centro y fundamento de todo el poema, que simboliza el puro e ideal amor de Don Quijote, amor sin mezcla de la más leve concupiscencia, que ha sido concebido hacia una criatura imaginaria en la cual se simbolizan todos los seres delicados y débiles de la tierra y que Don Quijote no ha de reconocer cuando se le presente en forma de rústica labradora, declarando que por maleficio de malignos encantadores ha sido tan villanamente desfigurada la bella entre las bellas, Dulcinea del Toboso. El desarrollo de las precedentes ideas acompaña al buen caballero por el campo de Montiel en aquel abrasador día del mes de julio ${ }^{28}$.

La tercera exposición de la sección B incorpora una novedad melódica de relevancia para el conjunto de la obra, este pequeño motivo en el piccolo, al que llamamos motivo de llamada y que se repetirá en las sucesivas recuperaciones de esta sección.

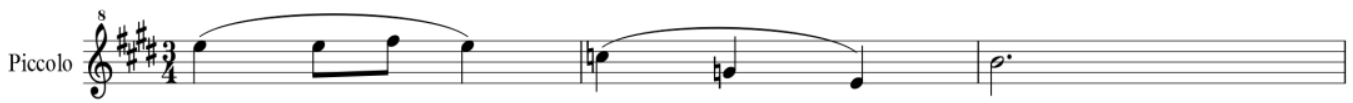

Ejemplo 3. Compases 307-308 del flautín del 1 ${ }^{\text {er }}$ canto de La primera salida de Don Quijote de la Mancha.

${ }^{28}$ Ibidem, pp. 5 y 6. 


\section{BeAtriz Alonso Pérez-Ávila}

El segundo canto comienza con la inscripción: "Vio no lejos del camino una venta" indicándonos en qué momento del capítulo II se encuentra don Quijote. Este canto no presenta una estructura claramente seccionada, como sí hizo en el primer canto, sino que se acerca a la Forma Sonata, careciendo de recapitulación y recuperando motivos y melodías ya presentados en el primer canto e incorporando otros nuevos.

El discurso dramático nos sitúa ante una serie de personajes populares que van a determinar el carácter tremendamente festivo y popular de este canto, en el que el plano contemplativo expuesto hasta este momento se ve relegado por otro más folklórico y dancístico. A pesar de eso, parte del contenido melódico es el mismo que el que encontramos en la primera parte; el motivo de llamada o el tema de Dulcinea de la sección $\mathrm{D}$ se reproducen.

La Introducción establece continuidad tonal (Mi Mayor) y melódica con el canto anterior, reproduciendo el motivo de llamada. 
LA CREACIÓN SINFÓNICA TRAS EL III CENTENARIO DEL QUIJOTE. EL COMPOSITOR EMILIO SERRANO Y SU OBRA DE TEMÁTICA CERVANTINA

\begin{tabular}{|c|c|c|c|c|c|c|}
\hline & Introducción & $\mathrm{A}$ & B & $\mathrm{C}$ & Desarrollo & Coda \\
\hline \multirow[t]{3}{*}{ Compases } & \multirow[t]{3}{*}{ cc. $1-20$} & cc. 21-45 & $\begin{array}{ll}\text { cc. } & 46- \\
60 & \end{array}$ & $\begin{array}{ll}\text { cc. } & 84- \\
114 & \end{array}$ & \multirow[t]{3}{*}{ cc. $123-200$} & \multirow[t]{3}{*}{ cc. 201-256, final } \\
\hline & & cc. $61-68$ & $\begin{array}{ll}\text { cc. } & 69- \\
83 & \end{array}$ & \multirow[t]{2}{*}{$\begin{array}{l}\text { cc. 119- } \\
122\end{array}$} & & \\
\hline & & & $\begin{array}{l}\text { cc. } 115- \\
118\end{array}$ & & & \\
\hline \multirow[t]{3}{*}{ Tonalidades } & \multirow[t]{3}{*}{ Mi M, Do m } & $\begin{array}{l}\text { Do M, Fa } \\
\text { M, Re M, } \\
\text { La m, Fa } \\
\text { M, Mi M }\end{array}$ & $\begin{array}{l}\mathrm{La} \text { M, } \\
\mathrm{Fa \#} \text { m }\end{array}$ & \multirow[t]{2}{*}{$\begin{array}{l}\text { La m, Re } \\
\text { m, Fa M, } \\
\text { Lab M, } \\
\text { Reb M, } \\
\text { Fa m }\end{array}$} & \multirow{3}{*}{$\begin{array}{l}\text { La M, Fa\# } \\
\text { m, Re M, } \\
\text { La M, Fa } \\
\text { M, Do M, } \\
\text { Sol M, La } \\
\text { m, Re m, } \\
\text { Do M, Sol } \\
\text { M, Do M, } \\
\text { La M, Do } \\
\text { M, La M, } \\
\text { La m, Sib } \\
\text { M, }\end{array}$} & \multirow[t]{3}{*}{$\begin{array}{l}\text { Fa M, Fa m, Lab } \\
\text { M, Fa m. }\end{array}$} \\
\hline & & \multirow[t]{2}{*}{ Solb M } & La m & & & \\
\hline & & & $\begin{array}{l}\mathrm{Fa} \mathrm{m}, \\
\text { Lab M }\end{array}$ & $\begin{array}{l}\text { Do M, } \\
\text { Fa M }\end{array}$ & & \\
\hline \multirow[t]{3}{*}{ Compás } & \multirow[t]{3}{*}{$3 / 4,4 / 4$} & $\begin{array}{l}6 / 8,2 / 2, \\
6 / 8\end{array}$ & $6 / 8$ & \multirow[t]{2}{*}{$\begin{array}{l}4 / 4, \\
2 / 2,4 / 4\end{array}$} & \multirow[t]{3}{*}{$2 / 2$} & \multirow[t]{3}{*}{$\begin{array}{l}6 / 8, \quad 3 / 4, \quad 2 / 2, \\
4 / 4\end{array}$} \\
\hline & & \multirow[t]{2}{*}{$6 / 8$} & $6 / 8$ & & & \\
\hline & & & $6 / 8$ & $4 / 4$ & & \\
\hline \multirow{3}{*}{ Tempi } & \multirow{3}{*}{ Andante } & \multirow{3}{*}{ Moderato ${ }^{29}$} & $\begin{array}{l}\text { Piu } \\
\text { mosso. }\end{array}$ & $\begin{array}{l}\text { Sin } \\
\text { indicar }^{30}\end{array}$ & \multirow{3}{*}{$\begin{array}{l}\text { Pin [mosso], } \\
\text { Allegro }\end{array}$} & \multirow{3}{*}{$\begin{array}{l}\text { Andante }{ }^{31} \text {, Presto } \\
\text { ma non tanto, Primo } \\
\text { tempo, Andante. }\end{array}$} \\
\hline & & & & & & \\
\hline & & & Allegretto & $\begin{array}{l}\text { Primo } \\
\text { tempo }\end{array}$ & & \\
\hline
\end{tabular}

Tabla 4. Estructura general del $2^{\circ}$ canto de La primera salida de Don Quijote de la Mancha.

${ }^{29}$ En la versión orquestal [QJ 73] esta sección se señala como Allegretto.

${ }^{30}$ En la versión orquestal [QJ 73] la presentación de la sección C se señala como Tranquilo y Moderato.

${ }^{31}$ En la versión orquestal [QJ 73] el inicio de la Coda se señala como Moderato y Maestoso antes de cambiar a Presto ma non tanto para, finalmente, acabar en Maestoso assai. 


\section{BeAtriz Alonso PÉrez-Ávila}

\section{SECCIÓN A}

Está formada por una melodía de carácter popular llamada "Canto del Arriero", en Do Mayor y 6/8. Esta frase de 9 compases [5 + 4] y será el material melódico más representativo de todo el canto.

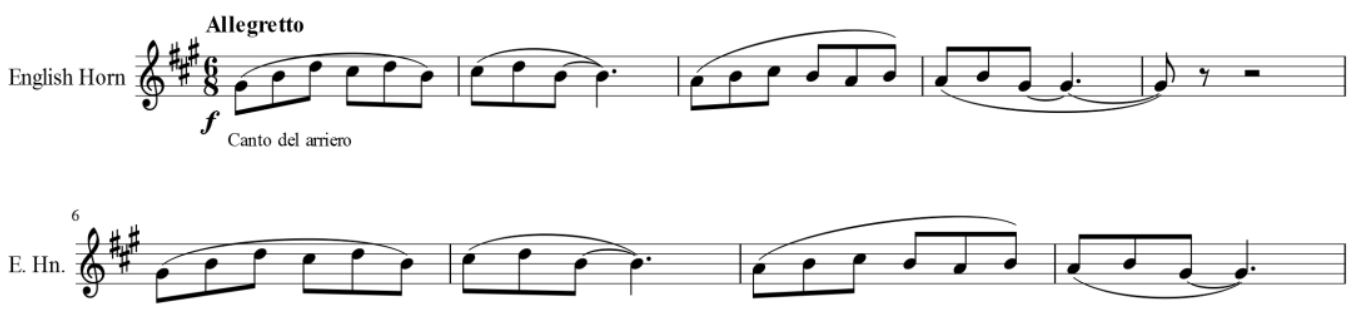

Ejemplo musical 4. Compases 21-29 del Corno Inglés del $2^{\circ}$ canto de La primera salida de Don Quijote de la Mancha.

La llegada de Don Quijote a la venta, su conversación con las damas del partido y con el ventero, el cuerno del porquero, el silbato del castrador, todo está encuadrado en un ambiente popular, muy característico de la personalidad artística del maestro Serrano. Es notable la idealización del motivo que dice primero el corno inglés, en do mayor, ingenuamente, con sencillez aldeana, que después adquiere tintas luminosas al ser repetido por la cuerda en mi mayor y que por fin se oye, ya idealizado, en la poética tonalidad de sol bemol: el ventero se ha convertido en castellano, las mozas de partido en damas, la venta en castillo, el silbato del castrador en música regalada, digna de ser oída en banquetes de príncipes (Subirá, [s.f.]: 5-6).

El "Canto del arriero" es, en primera instancia y como señala el programa de mano, muy sencillo y se sostiene sobre un bordón que incide en su carácter popular. En sucesivas apariciones se va a incorporar toda la orquesta aportando progresivamente riqueza tímbrica, armónica y rítmica.

\section{SECCIÓN B}

Tras el "Canto del arriero" y partiendo melódicamente del mismo, aparece otra sección de marcado carácter español, la cual surge del siguiente motivo interpretado por los violines: 
LA CREACIÓN SINFÓNICA TRAS EL III CENTENARIO DEL QUIJOTE. EL COMPOSITOR EMILIO SERRANO Y SU OBRA DE TEMÁTICA CERVANTINA

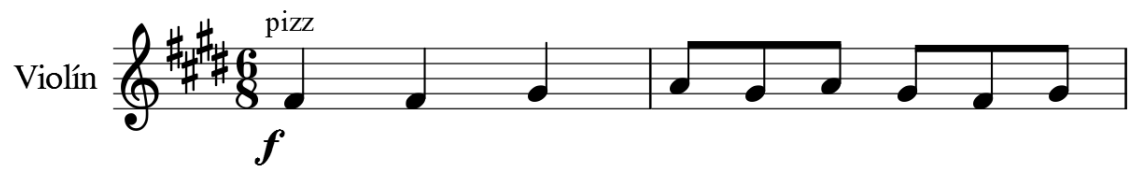

Ejemplo musical 5. Compases 45-46 del violín I del 2 canto de La primera salida de Don Quijote de la Mancha.

Este motivo contrapone las corcheas en $6 / 8$ con otro motivo en $3 / 4$, marcando una hemiolia horizontal en pizzicati que será característica de la sección B. Este nuevo motivo también se confrontará al "Canto del arriero"

\section{SECCIÓN C}

Súbitamente interrumpe Serrano esta imaginativa locura, que bien podría ser, siguiendo el programa, como se imagina Serrano el palacio y sus doncellas. El cambio es provocado por la exposición del material melódico principal de la sección $\mathrm{D}$ del primer canto, el tema de Dulcinea en un solo de los violonchelos acompañado simplemente por la cuerda y arpa, sobre La menor. Esta sección se caracteriza por las hemiolias horizontales y las recuperaciones melódicas de los dos cantos.

\section{DESARROLLO Y CODA}

Partiendo del "Canto del arriero", por aumentación, nos encontramos ante un pasaje Allegro sobre el que se va a incorporar toda la orquesta con los típicos procedimientos armónicos de los desarrollos clásicos, aumentando el ritmo armónico.

La coda de este poema sinfónico está construida sobre el material melódico que se ha expuesto a lo largo del mismo. En ella observamos con grandiosidad el recurso compositivo más característico de la obra de Serrano, la obsesión cíclica, presente a lo largo de toda la obra, le da unidad y coherencia narrativa al poema, pero en las secciones finales de las piezas sinfónicas de Serrano se convierte en motor y seña de identidad de este compositor. Las últimas recuperaciones melódicas provienen del inicio del $1^{\text {er }}$ Canto, recordando así Serrano que la marcha del caballero no se detiene, salvo para llamar a Dulcinea.

En relación con el capítulo II del Quijote, parece claro que Serrano intentó realizar una descripción fidedigna. La segunda parte del poema muestra la contraposición entre la realidad y el mundo que imagina don Quijote, teniendo siempre presente a su amada Dulcinea y toda su virtud ensoñadora, representada por ese tema romántico con el que finaliza -Ejemplo 2-. Interpretando las palabras del programa de concierto en relación a la partitura, el mundo real, popular, se representa por compases y ritmos binarios, que se 


\section{BeAtriz Alonso Pérez-Ávila}

contraponen a ritmos ternarios propios de ambientes cortesanos, su mundo exótico e idealizado de las caballerías.

\subsection{DON Quijote, CANTO SEGUNDO: LOS MOLINOS DE VIENTO}

Este poema sinfónico también comienza con una inscripción programática: "Mire Vuestra Merced, respondió Sancho, que aquellos que allí se parecen no son gigantes, sino molinos de viento...”. Esta famosa declaración de Sancho Panza nos lleva al capítulo VIII de la primera parte del Quijote.

\begin{tabular}{|c|c|c|c|c|c|}
\hline & Compases & Tonalidades & $\begin{array}{l}\text { Indicación de } \\
\text { compás }\end{array}$ & Tempi & $\begin{array}{l}\text { Material } \\
\text { motívico de La } \\
\text { primera salida de } \\
\text { Don Quijote }\end{array}$ \\
\hline \multirow[t]{3}{*}{ A } & cc. $1-15$ & Re m & $6 / 8,2 / 4$ & $\begin{array}{l}\text { Moderato quasi } \\
\text { Allegro }\end{array}$ & \\
\hline & cc. 37- 49 & Sol m & $6 / 8,2 / 4$ & $\begin{array}{l}\text { Moderato quasi } \\
\text { Allegro }\end{array}$ & \\
\hline & cc. $104-148$ & $\begin{array}{l}\text { Re M, Mib M, Sib } \\
\text { M, Re m }\end{array}$ & $6 / 8,2 / 4$ & $\begin{array}{l}\text { Moderato quasi } \\
\text { Allegro }\end{array}$ & Dulcinea \\
\hline \multirow[t]{3}{*}{$\mathrm{B}$} & cc. $16-36$ & Sol m, Re M & $6 / 8$ & & Canto del arriero \\
\hline & cc. $50-72$ & Re m, La M, Re m & $6 / 8$ & & Canto del arriero \\
\hline & cc. $149-195$ & $\begin{array}{l}\text { Re m, Sol M, Sol } \\
\text { m }\end{array}$ & $6 / 8$ & Andante & Canto del arriero \\
\hline $\mathrm{C}$ & cc. $73-83$ & Re m & $6 / 8$ & Allegro moderato & Dulcinea \\
\hline $\mathrm{D}$ & cc. $84-103$ & $\begin{array}{l}\text { Sol m, Solb M, Re } \\
\text { M. }\end{array}$ & $2 / 2$ & Moderato & $\begin{array}{l}\text { Trompas de la } \\
\text { sección C del } 1^{\text {er }} \\
\text { canto }\end{array}$ \\
\hline
\end{tabular}

Tabla 5. Estructura general de Don Quijote, Canto segundo: Los molinos de viento.

La forma de este poema sinfónico es muy próxima a la Forma Sonata, con la recapitulación de las secciones A y B. Además, el compositor reutiliza nuevamente elementos melódicos fundamentales del anterior poema sinfónico, el canto del arriero y el tema de Dulcinea. La sección $\mathrm{D}$ recupera el tema del maestoso del $1^{\text {er }}$ canto además de recurrir nuevamente a las hemiolias de La primera salida de Don Quijote, posiblemente para ayudar a la identificación geográfica de la obra. 
LA CREACIÓN SINFÓNICA TRAS EL III CENTENARIO DEL QUIJOTE.

EL COMPOSITOR EMILIO SERRANO Y SU OBRA DE TEMÁTICA CERVANTINA

La sección A es ligera, caracterizada por unos arpegios que se repiten insistentemente en unísono por prácticamente toda la orquesta. Este motivo, recurrente a lo largo del poema, parece que identifica las aspas de los molinos de viento que con tanta claridad ve Sancho Panza.

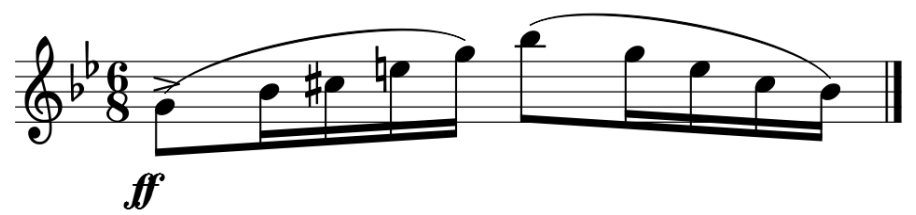

Ejemplo musical 6. Compás 1 del violín I de Don Quijote, Canto segundo: Los molinos de viento ${ }^{32}$.

El segundo material melódico fundamental de la sección A lo constituye una escala ascendente, de 8 notas, sobre la sensible de Re m, que utiliza también el séptimo grado rebajado. Esta contraposición a los arpegios iniciales, en 2/4, podría sugerir a los gigantes de Don Quijote. Así pues, nuevamente Serrano contrapone dos planos melódicos; uno, relacionado con la ensoñación quijotesca y el otro directamente sanchiano.

La sección B está articulada alrededor del "Canto del arriero", el cual es desarrollado más profundamente que en el anterior poema sinfónico. La sección $\mathrm{C}$, armónicamente estática, introduce por primera vez el recuerdo de Dulcinea, puesto que en este pasaje de la novela Don Quijote no duerme mientras piensa en su amada. La sección D recupera el tema del Maestoso de La primera salida de Don Quijote.

La última exposición de A nos devuelve a los molinos de viento, sobre los que emerge el clarinete para realizar cantando románticamente a Dulcinea. Finalmente, la sección B, nos trae de nuevo el "Canto del arriero", enfatizando su carácter popular, para finalizar con una pequeña coda en la que, la orquesta al unísono recuerda a Dulcinea, puesto que el Quijote siempre clama por su amada ante las alegrías y las penurias, como en el episodio con el vizcaíno de este capítulo VIII.

Armónicamente esta pieza es mucho más sencilla que el anterior poema, aunque ambas obras ofrecen el lenguaje romántico caracterizado por el uso de séptimas disminuidas, $\sigma^{a}$ aumentadas y una tendencia en la primera sección del primer poema sinfónico a utilizar el III como dominante secundaria con una tríada aumentada que provoca efectos sonoros muy interesantes. Serrano utiliza con frecuencia las notas pedales y los bordones, subrayando el carácter popular de la obra.

32 Edición propia partiendo de la fuente presente en la Biblioteca de la Fundación Juan March de Madrid [Sig. M-416-A]. 


\section{BeAtriz Alonso PÉrez-Ávila}

En los dos poemas sinfónicos destaca el tratamiento tímbrico que realiza Serrano, contraponiendo los dos focos orquestales: la cuerda y el viento madera, al que acompaña el peculiar uso solista de instrumentos de viento madera sobre fondos sonoros, característica que repetirá en su última obra sinfónica, la Elegía a la muerte de Fernando Villamil (1915).

\section{CONTROVERSIAS EN TORNO A LOS DOS POEMAS SINFÓNICOS}

Para finalizar, consideramos importante señalar que, en estudios anteriores al aquí presentado, se ha perpetuado una confusión sobre las obras de inspiración quijotesca de Emilio Serrano, puesto que se ha señalado que, además de estos dos poemas sinfónicos, existe un tercero denominado Don Quijote de La Mancha, el cual algunos estudios (Siemens, 2010: 285) datan en 1920. Consideramos que el error proviene de la biblioteca de Víctor Espinós. En la Biblioteca de Música Municipal de Madrid La primera salida de Don Quijote de La Mancha está denominada simplemente Don Quijote y fechada en 1920, aunque en ningún cartel, programa, partitura o crítica se la denominase de esta manera o se le atribuyese esa fecha. Este dato lo repite el propio Espinós en su estudio El Quijote en la música (Espinós, 1947: 29-30). Hay que recordar que Serrano escribió los poemas sinfónicos anteriormente referidos en los años que abarcan el período entre 1905 y 1908. Sin embargo, sí es cierto que en Don Quijote de La Mancha. Canto segundo: los molinos de viento, se reutilizan melodías y motivos rítmicos que aparecen en La primera salida de Don Quijote de La Mancha. Esto ha llevado a decir que el primero de ellos es, en realidad, una "refundición" de las ideas que tenía Serrano para los dos poemas (Ruiz Tarazona, 2005).

En la biografía del compositor encontramos detalles sobre La primera salida, pero Subirá apenas repara en el segundo poema sinfónico. Se limita a señalar su existencia, datar la fecha de composición y no recoge ninguna opinión de Serrano sobre su intención. Una explicación podría ser que, efectivamente, realizase apuntes para Los molinos de viento e incorporase algunas ideas que le gustaron para el poema que estaba acabando, que era $L a$ primera salida de Don Quijote.

Las características musicales de ambos poemas son semejantes, ambos contienen recursos estereotipadamente españoles que se sintetizan en el uso de ciertos compases $-6 / 8$ y 3/4 las hemiolias, escalas sobre La y, sobre todo, un uso muy significativo del tercer grado, así como de los giros melódicos sobre Mi, aunque Serrano parece muy consciente de que las sonoridades "andalucistas" no deben estar en la obra cervantina, con lo que ni encontramos cadencias andaluzas ni ritmos que nos acerquen a los tópicos más típicos del nacionalismo pintoresquista. Las dos obras tienen características musicales semejantes porque pertenecen al mismo período compositivo del autor. Sin embargo, parece que el lenguaje compositivo está más acabado en el primero de los dos poemas, pese a que habría que elaborar dos ediciones críticas para que ambos pudieran ser comparados.

La confusión es notoria; por un lado, tenemos un poema sinfónico estrenado e interpretado en diversas ocasiones a lo largo de más de quince años y, por el otro, tenemos un poema sinfónico que apenas se intentó estrenar. El primer poema está dividido en dos partes y la segunda de ellas formalmente es muy semejante al segundo poema sinfónico no estrenado. ¿Sería su intención escribir uno solo? ¿Querría sustituir el segundo canto del primer poema por Los Molinos de Viento?. Pero, en ese caso, ¿por qué Serrano sitúa los 
LA CREACIÓN SINFÓNICA TRAS EL III CENTENARIO DEL QUIJOTE.

EL COMPOSITOR EMILIO SERRANO Y SU OBRA DE TEMÁTICA CERVANTINA

poemas sinfónicos en diferentes capítulos de la novela? A pesar de las dudas que genera el hecho de que los segundos cantos pudiesen ser intercambiables, las inscripciones programáticas parecen indicar que, realmente, la intención de Serrano era describir episodios distintos y, por tanto, dos poemas sinfónicos diferenciados. Serrano reutiliza melodías porque para él tienen la misma significación. La recuperación de material melódico, en realidad, responde más a la obsesión cíclica de Serrano y a la significación programática de los temas. El "Canto del arriero", es, efectivamente, un tema que identifica a los arrieros y otras figuras populares. El tema romántico se relaciona con el amor, con Dulcinea; y, finalmente, el tema del maestoso representa al hidalgo caminando con determinación hacia un objetivo. El material melódico y el estilo compositivo es semejante, pero el discurso narrativo es diferente. Son dos obras distintas del mismo período creativo de Serrano que han tenido una vida dispar. Una, estrenada y celebrada y, la otra, olvidada en un archivo.

\section{BIBLIOGRAFÍA}

Alonso Pérez-Ávila, B. (2015). El compositor Emilio Serrano y Ruiz. (1850-1939): su compromiso regeneracionista con las instituciones del Madrid coetáneo. Estudio analítico de su producción instrumentaly sinfónica (tesis doctoral no publicada). Oviedo: Universidad de Oviedo.

Cortizo Rodríguez, M. E. (1990). "Emilio Serrano a los cincuenta años de su muerte". Cuadernos de música y teatro, Vol. 9, pp. 95-104.

Cuadrado Caparrós, M. D. (2010). "La recepción de la figura de don Quijote en la Orquesta Filarmónica de Madrid entre 1915-1936)", en B. Lolo (ed.). Visiones del Quijote en la música del siglo XX, pp. 253-276. Madrid: Centro de Estudios CervantinosMEC.

Espinós, V. (1947). El Quijote en la música. Barcelona: CSIC, Patronato del IV Centenario del Nacimiento de Cervantes.

García Álvarez de la Villa, B. (2016). "El Instituto Filarmónico del Conde de Morphy y su Escuela de Canto en el establecimiento del drama lírico nacional". Cuadernos de música iberoamericana, 29, pp. 81-109.

Gracia Iberni, L. (1995). Ruperto Chapí. Madrid: ICCMU. 


\section{BeAtriz Alonso Pérez-Ávila}

Lolo, B. (2010). "Interpretaciones del ideal cervantino en la música española del siglo XX (1905-1925)", en B. Lolo (ed.), Visiones del Quijote en la música del siglo XX, pp. 83-107. Madrid: Centro de Estudios Cervantinos-MEC.

Manrique de Lara, M. (1915, 23 de mayo). "Emilio Serrano”. Blanco y Negro, p. 36.

Martínez del Fresno, B. (1999). Julio Gómez: Una época de la música española. Madrid: ICCMU.

Nommick, Y. (2007). "El Quijote en la música del siglo XX: metamorfosis, fantasías y nuevas visiones", en B. Lolo (ed.), Cervantes y el Quijote en la música. Estudios sobre la recepción de un mito, pp. 209-239. Madrid: Centro de Estudios Cervantinos-MEC.

Presas Villalba, A. (2007). “1905: La trascendencia musical del III Centenario”, en B. Lolo (ed.), Cervantes y el Quijote en la música. Estudios sobre la recepción de un mito, pp. 285-305. Madrid: Centro de Estudios Cervantinos-MEC.

Sánchez, V. (2002). Tomás Bretón. Un músico de la Restauración. Madrid: ICCMU.

Serrano Ruiz, E. (1901). Estado actual de la música en el teatro, discurso leído en la recepción pública de Emilio Serrano y Ruir el día 3 de noviembre de 1901; y contestación de Ildefonso Jimeno de Lerma. Madrid: Imprenta de la Revista de Legislación.

Siemens, L. (2010). "El Quijote en la música orquestal del siglo XX", en B. Lolo (ed.): Visiones del Quijote en la música del siglo XX, pp. 279-295. Madrid: Centro de Estudios Cervantinos-MEC, 2010. p.285.

Ruiz Tarazona, A. (2005). "El Quijote en la música sinfónica”. En Álvarez Cañibano A. (Coord), El Quijote y la música [publicación virtual]. Madrid: Instituto Cervantes, Centro de Documentación de Música y Danza - INAEM, Recuperado de: http://cvc.cervantes.es/ACTCULT/quijote musica/ruiz.htm

Turgueniev, I. (2008). Hamlety Don Quijote (Javier Eraso, trad.). Madrid: Seguitor.

Fecha de recepción: 15/12/2016

Fecha de aceptación: 10/03/2017 